\title{
A Comparison of Absorbed Dose Determinations in Graphite by Cavity lonization Measurements and by Calorimetry*
}

\author{
Ben Petree and Paul Lamperti
}

\author{
Institute for Basic Standards, National Bureau of Standards, Washington, D.C. 20234
}

(September 27, 1966)

\begin{abstract}
The absorbed dose in graphite from a cobalt-60 gamma-ray source was measured with a spherical air-filled cavity ionization chamber and with two spherical calorimeters. The instruments were constructed from high-purity graphite. The current per unit mass of air and the absorbed power per unit mass of graphite were determined with uncertainties of 0.40 and 0.17 percent respectively. When the two results are combined the value of $W_{\text {air }} \cdot \bar{s}_{m}$ is found to be 33.72 electron volts in graphite per ion pair in air, with an uncertainty of \pm 0.14 electron volts.
\end{abstract}

Key Words: Absorbed dose, calorimeter, cavity ionization chamber, cobalt-60 gamma rays, comparison, graphite, $W \cdot \bar{s}_{m}$.

\section{Introduction}

Ionization techniques are often employed for the determination of radiation exposure and absorbed dose. Especially for photons in the megavolt region, the instrument of choice is a cavity ionization chamber. For absolute determinations of exposure with such a device the effective stopping power ratio, $\bar{s}_{m}$, contributes the largest single uncertainty-about 0.5 to 2 percent [1]. ${ }^{1}$ For absorbed dose determinations with a cavity chamber the principal contributors to the uncertainty are the stopping power ratio and the value of $W$, the average energy required to produce an ion pair in the gas. For air, the uncertainty in $W$ is about 0.4 percent [1]. Higher accuracy for such determinations therefore necessitates a reexamination of the values of $W$ and $\bar{s}_{m}$. The present investigation in which measurements are made, both with the ionization technique and with the calorimetric technique of the same beam of cobalt- 60 gamma rays, provides data on $W \cdot \bar{s}_{m}$.

When the necessary conditions of homogeneity in the material and uniformity of primary radiation are satisfied the relation [2] between ionization and energy deposited is given by the Bragg-Gray equation:

$$
\frac{P}{M}=W \cdot \bar{s}_{m} \cdot \frac{I}{m}
$$

where $\frac{I}{m}$ is the ionization current produced by the radiation per unit mass of cavity gas and $\frac{P}{M}$ is the power deposited by the radiation per unit mass of the ab-

*This work was supported by the Division of Biology and Medicine of the United States Atomic Energy Commission.

${ }^{1}$ Figures in brackets indicate the literature references at the end of this paper. sorbing material. If $\frac{I}{m}$ and $\frac{P}{M}$ are given in amperes per gram of gas and watts per gram of absorbing material respectively, then $W$ is to be given in electron volts per ion pair. In the experiments to be described in this paper the values of $\frac{P}{M}$ and $\frac{I}{m}$ were measured with small graphite calorimeters and an air-filled graphite wall cavity ionization chamber respectively, using cobalt-60 gamma radiation. The quotient of the measured values of $\frac{P}{M}$ and $\frac{I}{m}$ constitutes an experimental determination of the effective value of the product $W \cdot \bar{s}_{m}$ in terms of mass and electrical units. Such pairs of measurements have been made most recently by Bewley [3] and previously by Bernier et al. [4], and by Reid and Johns [5]. The present experiment repeats work that was done earlier in this laboratory [6] but with improved procedures that result in increased accuracy.

\section{Apparatus}

\subsection{Radiation Source}

The source of radiation for these measurements was a cylindrical assembly of twelve encapsulated cobalt-60 pencils, each a half-inch in diameter and 6 in long, containing about $1000 \mathrm{Ci}$ (curies) in all. The pencils are mounted upright on a steel base plate and are spaced equally around a $4 \frac{1}{8}$ in diam circle of centers. This assembly is mounted near the bottom of a square concrete cistern $6 \mathrm{ft}$ on a side in the laboratory floor. The source is covered by water to a depth of $10 \mathrm{ft}$. The ion chamber and calorimeter were mounted in a watertight can and, during measurements, were placed at the geometric center of the 
source. The exposure rate at that point was about 55 roentgens per second.

The exposure rate is fairly uniform near the center of the source. For example, the average exposure rate within a spherical volume $1.5 \mathrm{~cm}$ in diameter, which is the approximate inside dimension of the instruments, is only about 0.01 percent greater than at the center.

The spectrum of photons inside the can was examined and found to have approximately the composition given in table 1 [7].

TABLE 1. Spectrum of cobalt-60 source

\begin{tabular}{|c|c|c|}
\hline Component & $\begin{array}{c}\text { Fraction } \\
\text { of } \\
\text { intensity }\end{array}$ & Energy \\
\hline Primary photons.............. & 0.80 & $1.17 \mathrm{MeV}$ and $1.33 \mathrm{MeV}$ \\
\hline Singly scattered photons...... & .18 & $0.21 \mathrm{MeV}$ to $1.33 \mathrm{MeV}$ \\
\hline Multiply scattered photons... & .02 & $0.105 \mathrm{MeV}$ (effective energy) \\
\hline
\end{tabular}

This description of the source is clearly not unique. For example, Ritz and Attix, studying a nearly identical source, found that the responses they obtained with a set of energy-dependent chambers could be adequately explained by assuming a scattered intensity of 1.7 percent at $169 \mathrm{keV}$ and a primary radiation of 98.3 percent [8].

\subsection{Calorimeter}

The two adiabatic calorimeters used in these measurements each comprise two main parts; a spherical calorimetric body, or core, and a spherical enclosing shell. They are similar to one previously described $[6,9]$. Figure 1 shows the essential dimensions and details of one of the present instruments. A thermo-

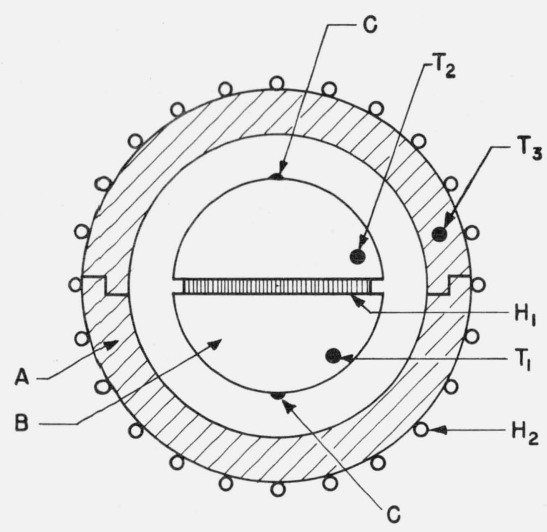

FIGURE 1. Spherical graphite calorimeter with solid core. A Shell: Outer diam, $2.05 \mathrm{~cm}$; inner diam, $1.28 \mathrm{~cm}$; graphite.

B Core: Diam, $1.00 \mathrm{~cm}$; graphite.

$T_{1}, T_{2}, T_{3}$, Thermocouples: Constantan versus chromel P; 0.003 in diam wires imbedded in holes with epoxy resin

$H_{1}$ Core heater made of electrically conducting resin.

$C$ Copper spots. A No. 43 copper wire was attached to each spot with epoxy resin.

$\mathrm{H}_{2}$ Shell heater. Karma resistance wire. Diam, 0.003 in. Attached to shell with epoxy resin. Space between wires is about $0.2 \mathrm{~cm}$. couple, $T_{1}$, is inserted in a shallow hole in the core and securely fastened. It is used to measure changes in core temperature with respect to reference junctions held at a constant temperature. Thermocouples $T_{2}$ and $T_{3}$, which are attached to the core and shell respectively, are connected in opposition and permit the measurement of temperature differences that may develop between the core and the shell during measurements.

Two cores differing in thickness were used in order to be able to determine whether or not the attenuation of the gamma rays within the core was properly evaluated. One core was made of solid hemispheres that were joined together by a film, $H_{1}$, of electrically conductive resin. The resistor thus formed measured about $100 \Omega$. It was used as a source of heat for calibrating changes in potential of $T_{1}$ in terms of energy input to the core. The second core was made of two hollow hemispheres having the same outer diameter as the first, but only about two-thirds the mass. These were joined in the same way as the first. The cores were carefully weighed at each step of assembly and the composition by weight of each component is given in table 2 .

TABLE 2. Composition by weight of calorimeter cores

\begin{tabular}{|c|c|c|c|c|}
\hline \multirow{2}{*}{ Component } & \multicolumn{2}{|c|}{ Solid core } & \multicolumn{2}{|c|}{ Hollow core } \\
\hline & Mass & Fraction & Mass & Fraction \\
\hline Graphite..... & $\begin{array}{l}\text { Gram } \\
0.9058_{0}\end{array}$ & 0.9945 & $\begin{array}{l}\text { Gram } \\
0.5946_{1}\end{array}$ & 0.9929 \\
\hline $\begin{array}{l}\text { Conducting } \\
\text { resin......... }\end{array}$ & $.0004_{x}$ & .0005 & $.0000_{1}$ & .0000 \\
\hline 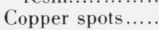 & $.0002_{0}$ & .0002 & $.0001_{7}$ & .0003 \\
\hline 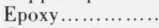 & $.0009_{1}$ & .0010 & $.0007_{\text {s }}$ & .0013 \\
\hline Copper wire...... & $.0002_{9}$ & .0003 & $.0001_{8}$ & .0003 \\
\hline Thermocouples.. & $.0031_{2}$ & .0034 & $.003 l_{1}$ & .0052 \\
\hline Total.... & $0.9108_{0}$ & 0.9999 & $0.5988_{6}$ & 1.0000 \\
\hline
\end{tabular}

The calorimeter shells were also made in halves that fit tightly together. The thickness was sufficient to stop electrons generated outside the shell by cobalt- 60 photons. Four hollow polystyrene spheres of about $1.5 \mathrm{~mm}$ diam were cemented to the inside surface of the shell to hold the core in the central position. An insulated wire heater, $\mathrm{H}_{2}$, was wound over the outer surface of the shells and attached with epoxy resin. An adjustable current generated heat in the winding so as to permit control of the shell temperature. Control was automatic during the measurements, and kept the shell temperature equal to that of the core.

The graphite of which the calorimeters and ion chamber were made was a reactor grade material, a sample of which left a residue of 0.014 percent when ashed in a muffle furnace. Calcium, vanadium, and titanium were the principal constituents detected by spectrographic analysis of the residue. This level of impurity has no significant effect upon the observed gamma-ray heating rates. The density of a sample of the graphite was $1.76 \mathrm{~g}$ per $\mathrm{cm}^{3}$. 

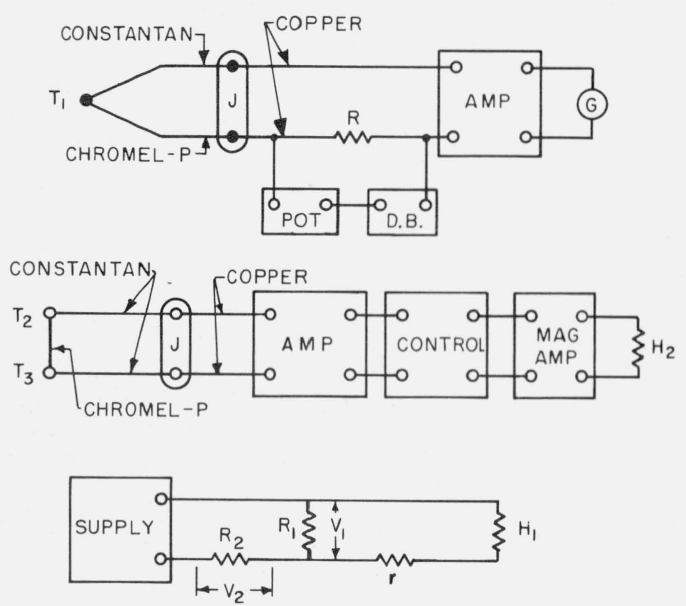

FIGURE 2. Electrical circuits used with calorimeters. (1) Core temperature measurement. $T_{1}$, core thermocouple; $J$, constant temperature reference cell; $R, 10 \Omega$ manganin standard; POT, 0-1.6 V potentiometer; D.B., 5 dial decade resistor, 0 to $111,110 \Omega$; AMP, contact modulated d-c amplifier; G, microammeter with zero at center of scale.

(2) Shell temperature control. $T_{2}$, core thermocouple; $T_{3}$, shell thermocouple: $J$, con: stant temperature reference cell; AMP, contact modulated d-c amplifier; CONTROL, 3-mode current adjusting controller: MAG AMP, magnetic amplifier; $H_{2}$, shell heater winding.

(3) Electrical calibration. SUPPLY, constant current generator; $R_{1}, R_{2}$, wirewound resistors: $r$, copper lead wires: $H_{1}$, core heater.

Three electrical circuits were required for operating the calorimeters. They are shown in figure 2 and functioned as follows:

(1) Core temperature measurement. The EMF of the thermocouple, $T_{1}$, attached to the core was measured by balancing it with an EMF developed by a simple Lindeck circuit consisting of a manganin $10 \Omega$ standard resistor, $R$, a five dial decade resistor and a potentiometer set at $0.3 \mathrm{~V}$. Adjustments in the decade resistance were made to provide a range of potentials of from about 45 to $175 \mu \mathrm{V}$ across $R$. The null detection system consisted of a contact modulated d-c amplifier and a galvanometer. The connections of the thermocouple wires to the copper lead wires at $J$ were kept at a steady reference temperature of $26.8^{\circ} \mathrm{C}$ by immersing them in oil in the central tube of a glass cell containing solidifying diphenyl ether [10].

(2) Shell temperature control. The EMF of the opposed thermocouple pair, $T_{2}$ and $T_{3}$, was amplified by a second contact modulated amplifier in order to obtain indications of temperature differences between the shell and the core. During normal calorimeter operation the EMF was kept equal to zero by the action of a three-mode controller and a magnetic amplifier that regulated the current flowing through the heater winding, $H_{2}$, of the shell. The calorimeters were operated a few degrees $\mathrm{C}$ above the temperature of the surroundings, so that the electrical heat supplied to the shell could be balanced by radiative and convective heat loss.

(3) Electrical calibration. This circuit provided power to the core heater, $H_{1}$, and so permitted calibration of the core temperature indications in terms of added energy. Since it was observed that the core resistance increased slightly as the temperature rose during a measurement, a power stabilizing arrangement was employed consisting of a constant current source, and a calibrated stabilizing resistance, $R_{1}$, shunting the heater. A calibrated resistor, $R_{2}$, was also included as shown. A calibrated potentiometer was used to measure the voltages, $V_{1}$ and $V_{2}$, that developed across $R_{1}$ and $R_{2}$. To give the desired stabilizing effect the resistance of $R_{1}$ was adjusted to be equal to that of the core heater, $H_{1}$; for convenience in measurement, the resistance of $R_{2}$ was adjusted to be one-half that of $R_{1}$, so that $V_{1}$ was nearly equal to $V_{2}$.

\subsection{Graphite Cavity Ionization Chamber}

The important dimensions and details of the cavity ionization chamber are shown in figure 3 . The wall thickness is equal to that of the calorimeter shells. The inner and outer surfaces of the chamber are spherical except at the end where the instrument is supported. The cylindrical collecting rod has a spherical enlargement at the end to reduce the electric field strength in that vicinity and thus make the field strength more uniform throughout the cavity.

To obtain the volume of the cavity in the graphite shell it was weighed both when empty and when filled with distilled water. The volume of the cavity was then determined as the quotient of the observed mass of added water by the density of water at the corresponding temperature. Water was excluded

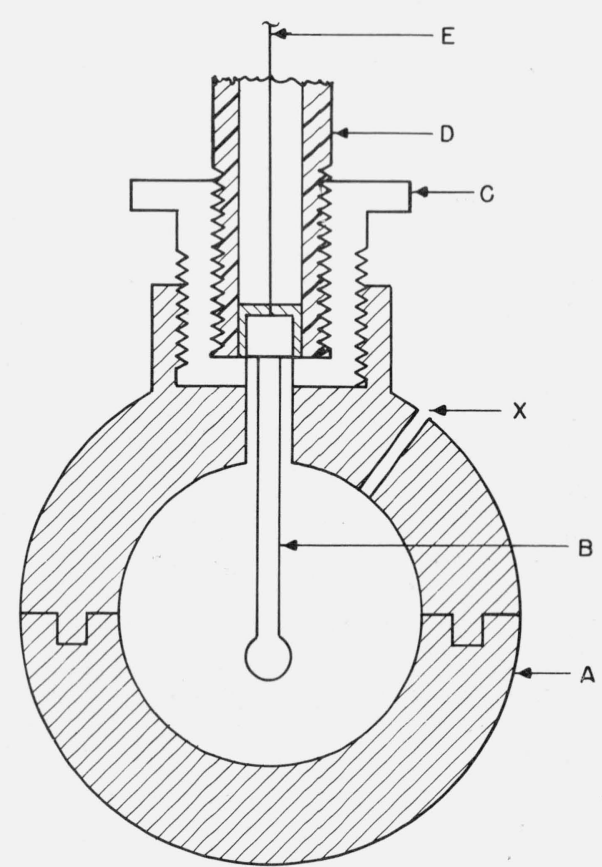

FIGURE 3. Spherical graphite wall cavity ionization chamber. A Graphite wall assembly. Outer diam, $2.07 \mathrm{~cm}$; inner diam, $1.27 \mathrm{~cm}$.

B Graphite collecting electrode. Diam, $0.1 \mathrm{~cm}$; diam of ball, $0.2 \mathrm{~cm}$; length, $1.1 \mathrm{~cm}$.

C Polystyrene high voltage insulator.

D Aluminum supporting stem with insulating plug to hold collecting electrode. Evacuated. E Current lead to electrometer.

$\begin{array}{lll}\mathrm{E} & \text { Current } \\ \mathrm{X} & \text { Air vent. }\end{array}$ 
from the air vent with a plug during the weighings. Corrections were made for atmospheric buoyancy acting on the added water. We believe there was no significant absorption of water into the graphite because the weight of the shell immediately after being emptied of water and wiped dry was not significantly different from the weight after the shell was kept overnight in vacuum.

Five determinations of the cavity volume were made, including one in which the cavity was filled with mercury rather than water. The results are given in table 3 .

TABLE 3. Volume of ionization chamber cavity

\begin{tabular}{|c|c|}
\hline Cavity filling & Computed volume \\
\hline Water. & $\begin{array}{c}c m^{3} \\
l^{2} 830\end{array}$ \\
\hline Marcury ......... & $\begin{array}{l}1.00373 \\
1.0773\end{array}$ \\
\hline Water........................ & 1.0770 \\
\hline Water........................ & 1.0771 \\
\hline Water.......... & 1.0800 \\
\hline Average............. & $1.07_{91} \pm 0.001_{3} a^{a}$ \\
\hline
\end{tabular}

${ }^{a}$ Standard error of the average.

The volume of the collecting electrode was calculated from its measured dimensions to be $0.0128 \mathrm{~cm}^{3}$. This was subtracted from the average determined above to give the volume of air within the assembled chamber.

The ionization current from the chamber was determined by measuring the corresponding voltage drop that it produced in flowing through a calibrated, wirewound resistor. A calibrated potentiometer was used for the voltage measurement, with a vibrating reed electrometer for null detection. The collecting voltage was supplied by dry batteries. An aneroid barometer was used to determine the air pressure. A mercury thermometer was used to measure the temperature of the surrounding water. The humidity of the air was shown to have no significant effect on the chamber current [11].

\section{Measuring Procedures}

\subsection{Calorimeter}

In both gamma-ray and electrical calibration runs the length of time required for the temperature of the calorimeter core to rise between two arbitrarily selected temperatures was determined. The temperatures corresponded to 160 and $60 \mu \mathrm{V}$ respectively, as indicated by the core thermocouple. Since the thermoelectric power of the measuring thermocouple was about $60 \mu \mathrm{V}$ per degree $\mathrm{C}$, the temperature interval amounted to about $1 / 3{ }^{\circ} \mathrm{C}$. The time required for the gamma rays to produce this change in temperature was about 40 min.

The final determination of gamma-ray heating power by this method does not require that the end points of the temperature interval be known, but only that they remain fixed during the set of runs. It was convenient, however, to divide the $100 \mu \mathrm{V}$ interval into fourths so that during electrical calibration runs five power observations could be made at nearly equal intervals of time.

A calibration run was made as follows: The temperature of the calorimeter was raised until a temperature indication of $175 \mu \mathrm{V}$ was attained, and at that time the automatic shell temperature control was turned on. Several observations, usually seven, of microvoltage were made at intervals of 5 min to determine an initial zero-power drift rate. The electrical calibrating power was then turned on. When a temperature indication of $160 \mu \mathrm{V}$ was attained (the controller having eliminated the transient shell-core temperature differential), an electronic timer was started, and measurements of $V_{1}$ and $V_{2}$ were made. $V_{1}$ and $V_{2}$ were also measured at $135,110,85$, and $60 \mu \mathrm{V}$. The timer was stopped at the $60 \mu \mathrm{V}$ indication. Calibrating power was turned off at $45 \mu \mathrm{V}$. Observations of microvoltage were then made at 5-min intervals as before, to determine a final zero-power drift rate. In reducing the data obtained in each run, the gross drift rate determined by the time interval between the 160 and $60 \mu \mathrm{V}$ indications was diminished by the average of the initial and final zeropower drift rates so as to obtain a corrected net rate.

For gamma-ray heating runs a similar schedule was followed. The calorimeter, placed near the corner of the pool where the radiation intensity was negligible, was brought to $175 \mu \mathrm{V}$ and the shell temperature was put under automatic control. Measurements of microvoltage were made for $30 \mathrm{~min}$ to determine the initial zero-power drift rate. The calorimeter was then inserted into the source. The timer was started when the temperature indication attained $160 \mu \mathrm{V}$, and was stopped when it attained $60 \mu \mathrm{V}$. At $45 \mu \mathrm{V}$ the calorimeter was removed from the radiation source to the corner of the pool and another set of measurements was made of microvoltage to determine the final zeropower drift rate. This measurement procedure was carried out with the calorimeter at different positions along the vertical axis of the source. Repeated measurements were then made at the position of maximum rate of temperature rise.

In this method of calorimetry a systematic error can result if the thermocouple pair used for automatic shell temperature control do not measure the average temperature of the core and shell surfaces to which they are attached. To evaluate this error the calibrations and gamma-ray measurements were done both with the calorimeters evacuated and open to normal atmospheric air. In addition, measurements of the time constants for the relaxation of core-shell temperature differentials with no shell heat were made under conditions of vacuum and atmospheric pressure.

\subsection{Cavity Chamber}

Preliminary measurements were made of chamber current versus chamber position along the vertical axis of the source to determine the position of maxi- 
mum response. At that position the current was measured over a range of collecting potentials up to $540 \mathrm{~V}$ to provide the data required for extrapolation to infinite field strength. To eliminate extra-cameral currents each current measurement was made with both positive and negative collecting potentials.

At the time of the measurement the atmospheric pressure was read from an aneroid barometer in the laboratory, and the temperature of the water was read from a mercury thermometer. The thermometer was brought to the surface for reading inside a l-gal bottle of water. The effect of scattering and attenuation in the chamber neck, insulator and stem was determined by measuring the chamber current with and without an equivalent dummy installed on the opposite side of the chamber.

\section{Results}

\subsection{Calorimetric}

The calibrations of the two instruments and the gamma-ray measurements obtained with them are given in table 4 . The gamma-ray measurements, which were made during a period extending from January to July 1965, have all been corrected to a common date, March 31, 1965, using 5.24 yr as the half-life of the radiation. The standard error shown with each mean value was computed from the deviations of that set of readings from its mean. It can be seen that the response of these instruments was, in each instance, less when it was operated at atmospheric pressure than in vacuum.

TABLE 4. Calorimeter measurements

\begin{tabular}{c|c|c|c}
\hline \hline \multicolumn{4}{c}{ (i) Calibrations, in microvolts per minute and milliwatt } \\
\hline \multicolumn{3}{c|}{ Solid core } & \multicolumn{2}{c}{ Hollow core } \\
\hline Vacuum & Air & Vacuum & Air \\
\hline 5.377 & 5.358 & 8.204 & 8.096 \\
5.380 & 5.357 & 8.180 & 8.120 \\
& 5.363 & 8.189 & 8.164 \\
& 5.346 & 8.202 & 8.138 \\
& 5.355 & & \\
& 5.344 & & \\
\hline Mean $5.376 \pm 0.003^{\text {a }}$ & 5.350 & & \\
\hline
\end{tabular}

(ii) Gamma-ray measurements, in microvolts per minute

\begin{tabular}{c|c|c|c}
\hline \multicolumn{2}{c|}{ Solid core } & \multicolumn{2}{c}{ Hollow core } \\
\hline Vacuum & Air & Vacuum & Air \\
\hline 2.509 & 2.507 & 2.548 & 2.497 \\
2.503 & 2.504 & 2.531 & 2.525 \\
2.520 & 2.491 & 2.544 & 2.549 \\
2.518 & 2.493 & 2.547 & 2.534 \\
2.522 & 2.509 & & \\
& 2.492 & Mean $2.544 \pm 0.003^{\text {a }}$ & $2.526 \pm 0.011^{\text {a }}$ \\
\hline
\end{tabular}

${ }^{\text {a }}$ Standard error of the mean.

The results of the temperature relaxation measurements are given in the four curves of figure 4 , and

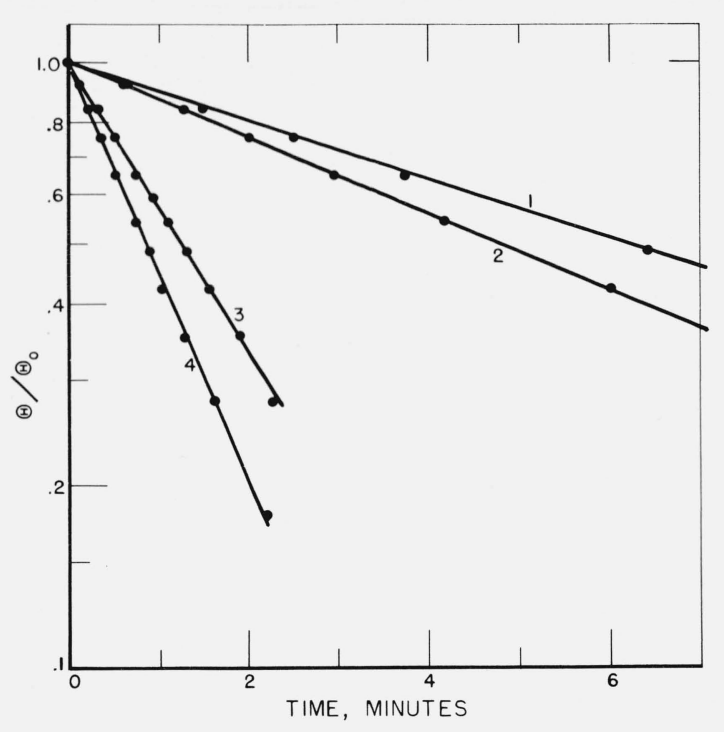

FiguRE 4. Relaxation of temperature differences between core and shell of calorimeter with no electrical shell heat.

$\theta / \theta_{0}$ is the fraction of the initial temperature difference. Initial temperature difference was produced by a pulse of heat in the core. Scale of ordinates is logarithmic. 1, solid core in vacuum; 2 , hollow core in vacuum; 3 , solid core in air; 4 , hollow core in air.

show clearly the importance of air as a heat conduction path between core and shell. The relaxation rates (reciprocal of time constant) determined from these data are 0.168 and 0.237 per minute respectively, for the solid and hollow core instruments in vacuum; 0.910 and 1.30 per minute respectively, for the solid and hollow core instruments in air. Since for each calorimeter this relaxation rate is $\lambda=k\left(\frac{1}{C_{1}}+\frac{1}{C_{2}}\right) \min ^{-1}$ where $k=$ core-shell heat transfer coefficient, joules per minute and ${ }^{\circ} \mathrm{C}, C_{1}=$ heat capacity of core, joules per ${ }^{\circ} \mathrm{C}$ and $C_{2}=$ heat capacity of shell, joules per ${ }^{\circ} \mathrm{C}$, the relaxation rates obtained above are proportional to the corresponding core-shell heat transfer coefficients.

As was noted from the mean values in table 4 , both the electrical and gamma-ray heating rates were affected by the addition of air with its consequent alteration of the core-shell heat transfer coefficient. The correct heating rate that is desired in both determinations is that which would be obtained with no heat transfer to the core. That rate may be arrived at by extrapolating linearly to zero, on a heat transfer scale, the two heating rates measured in air and in vacuum. The extrapolations are shown in figures $5 \mathrm{a}$ and $5 \mathrm{~b}$ and give the values shown in table 5 for the calorimetric results.

TABLE 5. Extrapolated calorimeter measurements

\begin{tabular}{c|c|c}
\hline \hline Calorimeter core & $\begin{array}{c}\text { Gamma-ray } \\
\text { heating rate }\end{array}$ & Calibration \\
\hline & $\mu V \min ^{-1}$ & $\mu V \min ^{-1} \mathrm{~mW}^{-1}$ \\
Solid......................................................... & 2.519 & 5.381 \\
Hollow.... & 2.548 & 8.213 \\
\hline
\end{tabular}



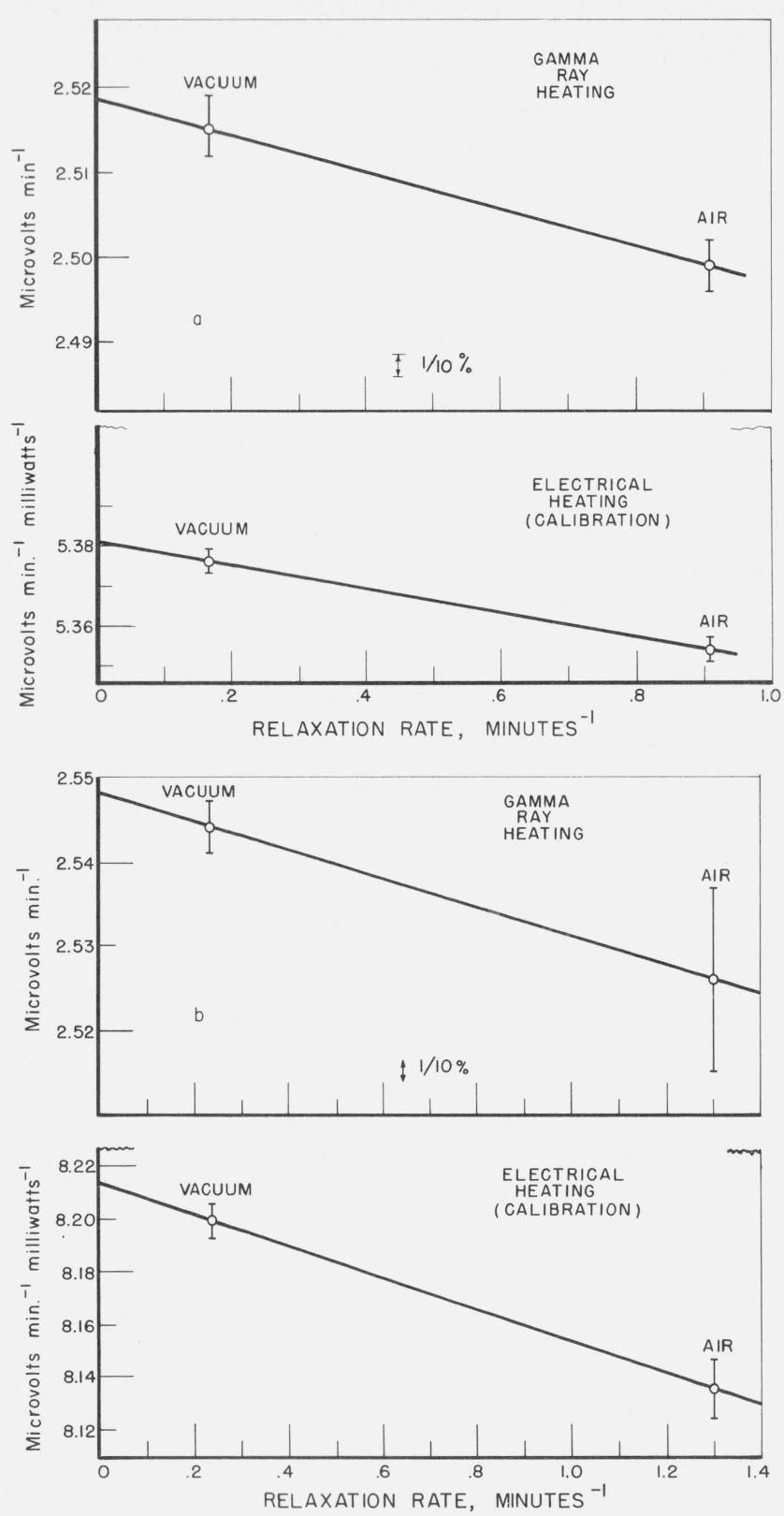

FIGURE 5. Variation of calorimeter response with core-shell heat transfer coefficient.

a, solid core; b, hollow core. The abscissae are proportional to the core-shell heat transfer coefficients.

From the values of the calorimetric results, shown in table 5 , and the masses given in table 2 , the gammaray heating powers per gram of the cores are obtained as follows:

(1) Solid Core: $\frac{P}{M}=2.519 \times \frac{10^{-3}}{5.381}$

$$
\times \frac{1}{0.9108}=0.5140 \times 10^{-3} W_{g^{-1}}
$$

(2) Hollow Core: $\frac{P}{M}=2.548 \times \frac{10^{-3}}{8.213}$

$$
\times \frac{1}{0.5989}=0.5180 \times 10^{-3} \mathrm{Wg}^{-1} .
$$

\subsection{Ionization Measurements}

Measurements of ionization current were made at the position of maximum observed response in the source on December 31, 1964, before the calorimetric measurements and on July 16, 20, 21, and 23, 1965, after their completion. The chamber currents observed at a series of collecting potentials were extrapolated to infinite field strength according to the method of Mie $[12,13]$ to determine the true ionization current in the absence of recombination. From extrapolations similar to those illustrated in figure 6 it was found that the collection efficiency at $540 \mathrm{~V}$ was about 0.993 . The measurements with and without the dummy stem showed that absorption in the stem reduced the observed current by 0.13 percent. Therefore, the observed currents were increased by the factor $\frac{1.0013}{0.993}$. The density of the air in the cavity chamber was computed using the expression

$$
\rho_{\text {air }}=0.001293 \frac{P}{760} \frac{273.2}{T} \mathrm{~g} / \mathrm{cm}^{3}
$$

where

$P=$ observed barometric pressure, $\mathrm{mm} \mathrm{Hg}$

$T=$ observed collecting volume air temperature ${ }^{\circ} \mathrm{K}$.

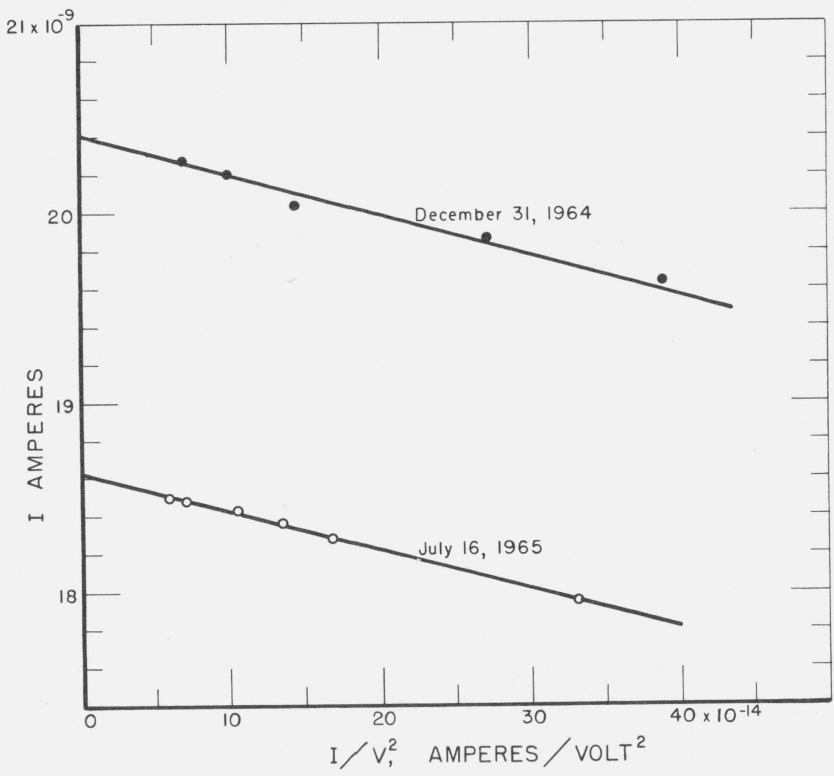

FIGURE 6. Extrapolation of observed chamber current to infinite field strength.

$I$, observed chamber current, amperes; $V$, collecting potential, volts. 
In the air density calculation, $0.4 \mathrm{~mm} \mathrm{Hg}$ was added to the observed pressure to allow for the difference in altitude between the barometer and the chamber, and $0.2{ }^{\circ} \mathrm{C}$ was added to the observed water temperature to allow for the gamma-ray heating of the chamber as measured in a separate experiment.

The mass of air was computed as the product of the volume of the cavity, $1.0663 \mathrm{~cm}^{3}$, and the density of the air at the time of measurement. The ionization current per unit mass of air was then determined. The observed currents and the results of the computations are shown in table 6 . The standard error of the average was computed from the deviations of the individual determinations from the average.

TABLE 6. Ionization measurements

\begin{tabular}{|c|c|c|c|c|c|c|c|}
\hline \multirow{2}{*}{$\begin{array}{c}\text { Date of } \\
\text { measure- } \\
\text { ment }\end{array}$} & \multicolumn{4}{|c|}{ Ionization current } & \multirow[b]{2}{*}{ Air density } & \multirow{2}{*}{ Mass of air } & \multirow{2}{*}{$\begin{array}{l}\text { Current } \\
\text { per unit } \\
\text { mass of air }\end{array}$} \\
\hline & Observed $^{a}$ & Corrected $^{b}$ & $\begin{array}{l}\text { Extrapo- } \\
\text { lated }^{\mathrm{c}}\end{array}$ & $\begin{array}{c}\text { Referred to } \\
3 / 31 / 65\end{array}$ & & & \\
\hline $12 / 31 / 64$ & $\begin{array}{c}\times 10^{-8} A \\
2.031_{5} \\
2.032_{4}\end{array}$ & $\begin{array}{c}\times 10^{-8} A_{4} \\
2.034_{1} \\
2.035_{0}\end{array}$ & $\begin{array}{l}\times 10^{-8} A \\
2.049_{2} \\
2.050_{1}\end{array}$ & $\begin{array}{c}\times 10^{-8} A \\
1.986_{0} \\
1.987_{0}\end{array}$ & $\begin{array}{c}\times 10^{-3} \mathrm{~g} / \mathrm{cm}^{3} \\
1.202_{\mathrm{s}} \\
1.202\end{array}$ & $\begin{array}{l}\times 10^{-3} \mathrm{~g} \\
1.282_{5} \\
1.282_{7}\end{array}$ & $\begin{array}{c}\times 10^{-5} . \mathrm{A} / \mathrm{g} \\
1.548_{\mathrm{s}} \\
1.549_{0}\end{array}$ \\
\hline $7 / 16 / 65$ & $\begin{array}{l}1.850_{1} \\
1.851_{i}\end{array}$ & $\begin{array}{l}1.852_{5} \\
1.853_{5}\end{array}$ & $\begin{array}{l}1.864_{5} \\
1.865_{6}\end{array}$ & $\begin{array}{l}1.940_{2} \\
1.941_{3}\end{array}$ & $\begin{array}{l}1.172_{3} \\
1.173_{8}\end{array}$ & $\begin{array}{l}1.250_{0} \\
1.251_{6}\end{array}$ & $\begin{array}{l}1.552_{2} \\
1.551_{1}\end{array}$ \\
\hline $7 / 20 / 65$ & $\begin{array}{l}1.862_{3} \\
1.858_{5}\end{array}$ & $\begin{array}{l}1.864_{8} \\
1.860_{9}\end{array}$ & $\begin{array}{l}1.876_{9} \\
1.873_{0}\end{array}$ & $\begin{array}{l}1.955_{5} \\
1.951_{5}\end{array}$ & $\begin{array}{l}1.180_{6} \\
1.179_{6}\end{array}$ & $\begin{array}{l}1.258_{\mathrm{g}} \\
1.257_{\mathrm{f}}\end{array}$ & $\begin{array}{l}1.553_{4} \\
1.551_{5}\end{array}$ \\
\hline $7 / 21 / 65$ & $\begin{array}{l}1.862_{8} \\
1.855_{0} \\
1.856_{6}\end{array}$ & $\begin{array}{l}1.865_{2} \\
1.857_{4} \\
1.859_{0}\end{array}$ & $\begin{array}{l}1.877_{3} \\
1.869_{5} \\
1.871_{1}\end{array}$ & $\begin{array}{l}1.956_{2} \\
1.948_{0} \\
1.949_{6}\end{array}$ & $\begin{array}{l}1.181_{6} \\
1.178_{2} \\
1.178_{0}\end{array}$ & $\begin{array}{l}1.259_{9} \\
1.256_{3} \\
1.256_{1}\end{array}$ & $\begin{array}{l}1.552_{7} \\
1.550_{6} \\
1.552_{1}\end{array}$ \\
\hline $7 / 23 / 65$ & $\begin{array}{l}1.843_{4} \\
1.838_{9}\end{array}$ & $\begin{array}{l}1.845_{8} \\
1.841_{3}\end{array}$ & $\begin{array}{l}1.857_{8} \\
1.853_{3}\end{array}$ & $\begin{array}{l}1.937_{7} \\
1.932,\end{array}$ & $\begin{array}{l}1.170_{5} \\
1.168_{5}\end{array}$ & $\begin{array}{l}1.248_{1} \\
1.246_{0}\end{array}$ & $\begin{array}{l}1.552_{3} \\
1.551_{3}\end{array}$ \\
\hline
\end{tabular}

Average $1.551_{4}$

At $540 \mathrm{~V}$ collecting potential.

Corrected for stem absorption.

' Extrapolated to infinite collecting potential.

d Standard error of the average.

\section{Analysis of Results}

\subsection{Correction of Calorimetric Measurements for Impurities and Core Attenuation}

In the relation

$$
W \cdot \bar{s}_{m}=\frac{\frac{P}{M}}{\frac{I}{m}},
$$

$\frac{I}{m}$ refers to the cavity chamber's response in amperes per gram of air in the cavity while $\frac{P}{M}$ refers to the calorimeter's response in watts per gram of carbon in the core. For this equation to hold accurately, corrections to the calorimetric results are required to allow for the radiation absorption of atoms other than carbon in the cores and for the effect of gamma-ray attenuation in the cores. No correction is needed for attentuation in the calorimeter shells as they were equal in thickness to the chamber wall, and the additional attenuation in the shell heater windings was negligible.

The effect of impurities is small and results in only a slight increase in gamma-ray heating relative to pure carbon. We calculate the excess, using the data of tables 1 and 2 and the mass energy transfer coefficients of Berger [14] to be 0.028 percent and 0.041 percent for the solid and hollow cores respectively.

In evaluating the core attenuation we have made use of data obtained by Loftus and Weaver [15] in this laboratory that show the variation with wall thickness of the response of a cavity ionization chamber. Three spherical chambers made of the same graphite as that used for the calorimeters were exposed in turn at the same distance from a cobalt-60 source similar in spectrum to the NBS water-shielded source. The resulting ionization current per gram of air within the cavity was determined for each chamber. The data of table 7 are the result of a series of such comparisons, all at source-chamber distances greater than $1 \mathrm{~m}$.

TABLE 7. Attenuation in graphite

\begin{tabular}{c|c|c|c}
\hline \hline Chamber & $\begin{array}{c}\text { Diameter } \\
\text { of cavity }\end{array}$ & $\begin{array}{c}\text { Thickness } \\
\text { of wall }\end{array}$ & $\begin{array}{c}\text { Current per gram of air } \\
\text { relative to chamber 2 }\end{array}$ \\
\hline & $c m$ & $m m$ & \\
1 & 1.588 & 2.41 & 1.0065 \\
2 & 1.270 & 4.00 & 1.0000 \\
3 & 0.953 & 5.585 & 0.9900 \\
\hline
\end{tabular}

These data indicate a fractional decrease in chamber response of 0.0051 per $\mathrm{mm}$ of wall thickness. Although the average wall thickness for each spherical chamber is larger than the radial thickness shown in table 7 , the differences of these averages were found not to be significantly larger than the radial difference. The linear attenuation is therefore correctly given as 0.0051 per $\mathrm{mm}$.

The length within the cores to be used for calculating the attenuation was obtained from a theorem of geometry due to Tomkeieff [16] that the average length $\bar{L}$ of the intercept in a spherical body is

$$
\begin{aligned}
\bar{L} & =4 \frac{V}{S} \text { where } \\
V & =\text { volume of sphere and } \\
S & =\text { surface area of sphere. }
\end{aligned}
$$

If the body contains voids and $V$ is taken to be the volume of the sphere less the volume of the voids, then the expression gives the average length of intercept not in a void. We find average gamma-ray paths in graphite from this theorem of $0.667 \mathrm{~cm}$ and $0.438 \mathrm{~cm}$ for the solid and hollow cores, respectively.

With no appreciable error of approximation the quotient of the average intensity of the radiation 
within the cores by the intensity outside can be taken to be

$$
\begin{aligned}
& 1-0.0051 \times \frac{6.67}{2}=0.9830 \text { for the solid core and } \\
& 1-0.0051 \times \frac{4.38}{2}=0.9888 \text { for the hollow core. }
\end{aligned}
$$

The absorbed gamma-ray power in watts per gram of pure carbon in the absence of core attenuation is therefore found to be by the two calorimeter determinations:

Solid core: $0.5140 \times \frac{1}{1.00028} \times \frac{1}{0.9830} \times 10^{-3}$

$=0.5227 \times 10^{-3}$ watts per gram.

Hollow core: $0.5180 \times \frac{1}{1.00041} \times \frac{1}{0.9888} \times 10^{-3}$

$=0.5237 \times 10^{-3}$ watts per gram.

Average: $0.5232 \times 10^{-3}$ watts per gram.

\subsection{Value of $\frac{P}{M} \div \frac{I}{m}$}

The average values of $\frac{P}{M}$ and $\frac{I}{m}$ determined in this work give for their quotient

$$
Q=\frac{\frac{P}{M}}{\frac{I}{m}}=\frac{0.5232 \times 10^{-3}}{1.551_{4} \times 10^{-5}}=33.72 \mathrm{eV}
$$

in carbon per ion pair in air.

\subsection{Accuracy of Result}

In estimating the uncertainty in the quotient $\frac{\frac{P}{M}}{\frac{I}{m}}$, we

have considered the effect of uncertainties in the determinations of mass, ion chamber current, electrical calibration power, the attenuation correction that was applied to the calorimetry, the field strength extrapolation of the ion chamber current and in the extrapolated values of the electrical calibration and gamma-ray rates.

The measurements of voltage in this experiment are subject to a systematic error of \pm 0.02 percent and a random reading error (standard error) of 0.01 percent which have been combined to give an overall uncertainty of 0.05 percent (three times 0.01 percent plus 0.02 percent).

The error in the ionization current measurements caused by an inability to null precisely is estimated to be no more than 0.04 percent.
The resistance value used in the ionization current measurements was considered to be accurate to within 0.01 percent. The values of $R_{1}$ and $R_{2}$ (fig. 2), used in the power calibration each have an uncertainty of not more than 0.05 percent.

An overall uncertainty of 0.13 percent has been assumed for temperature measurements, based on an estimated systematic error of 0.07 percent and a standard error of 0.02 percent, due to reading the instrument. No significant error is introduced by the method of measurement outlined above.

Pressure measurements are believed to be accurate to one part in 10,000 and are subject to reading errors of no more than 0.02 percent. These have been combined to give an overall uncertainty of 0.07 percent.

The uncertainty in the collection efficiency is estimated to be 0.06 percent, based on three times a standard error of 0.02 percent.

It has been estimated that the core attenuation correction introduces a systematic error of not more than 0.05 percent.

The mass of the calorimeter cores was believed to be known to two parts in 10,000 .

The uncertainty in the collecting volume of the cavity chamber has been estimated to be no more than three times the standard error of the mean value of the cavity chamber volume, from table 3 , since the error contributed by the collecting electrode volume measurement is negligible. Thus, an overall uncertainty of 0.36 percent has been assigned to the collecting volume.

Measurements before and after each of the two series of ionization measurements indicated that the chamber was off axis by only a small fraction of a millimeter. We estimate that this introduced no significant error in the ionization measurements.

The errors produced by the extrapolation process were computed to be no more than 0.10 percent and 0.04 percent for the gamma-ray heating and electrical calibration rates, respectively.

Tables 8 and 9 show the resulting uncertainties in $\frac{P}{M}$ and $\frac{I}{m}$, respectively, due to the different variables. The combined uncertainty in each ratio is the square root of the sum of squares of the individual uncertainties listed. The uncertainty in the value of $\frac{P}{M} \div \frac{I}{m}$ has been computed to be $(0.17+0.40)^{1 / 2}=0.43$ percent.

TABLE 8. Uncertainty in $\frac{\mathrm{P}}{\mathrm{M}}$

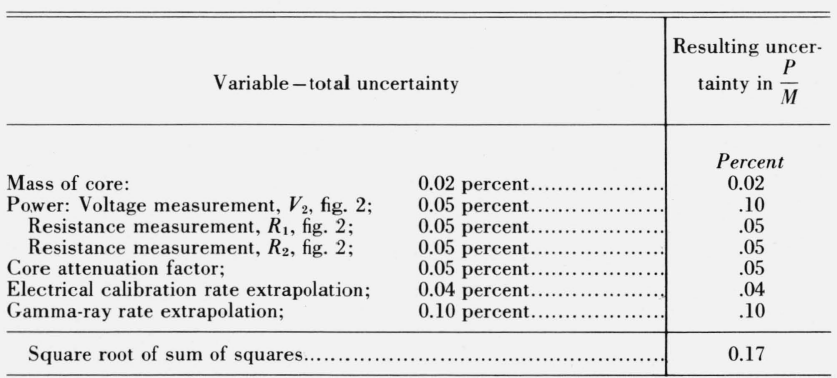


TABLE 9. Uncertainty in $\frac{\mathrm{I}}{\mathrm{m}}$

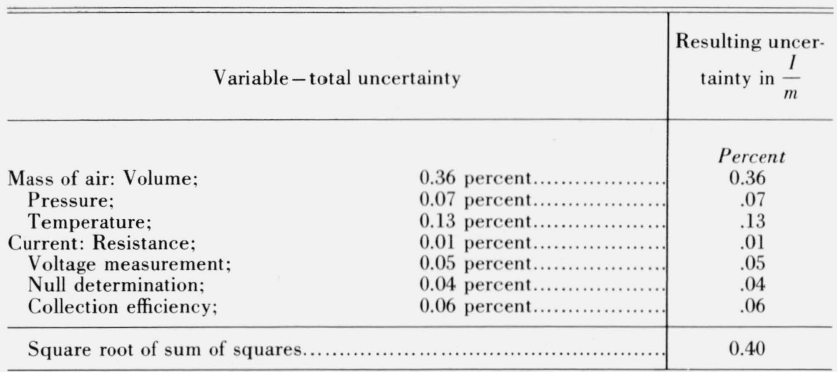

\subsection{Comparison With Other Measurements}

The previous measurements of this quotient that were mentioned in the introduction are shown in comparison to the present result in table 10 . These values should be directly comparable except for the slight differences in effective stopping power, $\bar{s}_{m}$, that may arise from the spectral dissimilarities of the different cobalt sources used. The values of $\bar{s}_{m}$ listed in table 10 are those given by the authors as applicable to their sources. The value for the NBS source was computed from the expression:

$$
\int \mu(E) i(E) d E \div \int \frac{1}{\bar{s}_{m}(E)} \mu(E) i(E) d E
$$

where $i(E)$ is the distribution in energy of photon intensity given in table $1, \mu(E)$ is the mass energy transfer coefficient of carbon given by Berger [14] and $\bar{s}_{m}(E)$ is interpolated from the mean mass stopping power ratios for graphite relative to air given in table IA7 of NBS Handbook 85 [1]. While the methods used for obtaining $\bar{s}_{m}$ are not entirely consistent with one another, the resulting discrepancies are evidently small and probably negligible in comparison to the experimental uncertainties.

The value of $Q$ included in table 10 from the earlier NBS measurements [6] differs from the other entries in that the value of ionization per unit mass of air was derived from an exposure rate measurement of the source with a calibrated chamber. The original data have been recomputed to remove a correction previously made for humidity and to change the attenuation correction so as to conform to that of the present experiment using the data of Loftus and Weaver. The uncertainty shown is largely that assigned to the NBS exposure standard in 1958.

The present measurements give the value of $Q$ and hence the value of the product $W \cdot \bar{s}_{m}$ with an uncertainty of about 0.43 percent. Since the uncertainty in each of the factors is about one-half percent, the effect of the present determination is to reduce the product's uncertainty by about a factor of two. Our estimates of error for the presently reported experiment are two-thirds and one-third those of Bewley and of Reid and Johns respectively.
Table 10 includes the quotient of $Q$ by $\bar{s}_{m}$ for each of the experiments. These should be equal, provided the values of $\bar{s}_{m}$ have been selected in a consistent way. The quotients show a reasonable degree of consistency, except for the early measurement of Bernier et al. If the data reduction computations of measurements 3 and 4 were to be altered to eliminate the humidity correction that was included, the quotients of $Q$ by $\bar{s}_{m}$ would be increased, although probably by no more than 0.1 percent.

The consistency of the values of $Q \div \bar{s}_{m}$ does not, however, imply a corresponding degree of accuracy of $W$, since each quotient carries with it the uncertainty in $\bar{s}_{m}$. The uncertainty in $\bar{s}_{m}$ does not appear to be easily reduced by direct experiment or computation.

A more accurate experimental determination of $W$, on the other hand, does appear to be possible, and would, if available, also provide a more accurate value for $\bar{s}_{m}$.

TABLE 10. Comparison of results

\begin{tabular}{|c|c|c|c|c|}
\hline $\begin{array}{c}\text { Measure- } \\
\text { ment } \\
\text { number }\end{array}$ & Authors & $Q=\frac{P}{M} \div \frac{I^{\mathrm{a}}}{m}$ & $\boldsymbol{s}_{m}{ }^{\mathrm{b}}$ & $W=Q \div \bar{s}_{m}{ }^{c}$ \\
\hline $\begin{array}{l}1 \\
2 \\
3 \\
4 \\
5\end{array}$ & 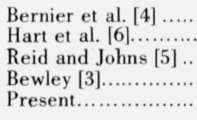 & $\begin{array}{l}33.07 \pm 0.21 \\
33.41 \pm 0.7 \\
34.15 \pm 0.31 \\
34.11 \pm 0.21 \\
33.72 \pm 0.14\end{array}$ & $\begin{array}{l}1.005 \\
1.004 \\
1.005 \\
1.004 \\
1.004\end{array}$ & $\begin{array}{l}32.91 \pm 0.21 \\
33.28 \pm 0.7 \\
33.98 \pm 0.31 \\
33.97 \pm 0.21 \\
33.59 \pm 0.14\end{array}$ \\
\hline
\end{tabular}

a Electron volts in carbon per ion pair in air.

${ }^{\mathrm{b}}$ Effective mass stopping power of carbon relative to air.

${ }^{\mathrm{c}}$ Electron volts in air per ion pair in air. Uncertainty does not include uncertainty in $\bar{s}_{m}$.

The authors are indebted to Harold O. Wyckoff for his continued interest in and many valuable discussions concerning this measurement.

\section{References}

[1] NBS Handbook 85, Physical Aspects of Irradiation (1964).

[2] L. H. Gray, Proc. Roy. Soc. (London), Al56, 578 (1936).

[3] D. K. Bewley, Brit. J. Radiol. 36, 865-878 (1963).

[4] J. P. Bernier, L. D. Skarsgard, D. V. Cormack, and H. E. Johns, Radiation Res. 5, 613-633 (1956).

[5] W. B. Reid and H. E. Johns, Raditition Res. 14, 1-16 (1961)

[6] E. J. Hart, H. W. Koch, B. Petree, J. H. Schulman, S. I. Taimuty, and H. O. Wyckoff, Proceedings of the second U.N. conference on the peaceful uses of atomic energy, Vol. 21, 189193 (1958), Geneva.

[7] B. Petree and J. C. Humphreys, Radiology 84, 129 (Jan. 1965).

[8] V. H. Ritz and F. H. Attix, Radiation Res. 16, 401-415 (1962).

[9] B. Petree and G. Ward, NBS Tech. Note 163 (1962).

[10] D. D. Enagonio, E. G. Pearson, and C. P. Saylor, Temperature, its measurement and control in science and industry, Vol. 3 , Part 1, 219-230 (Reinhold Publishing Corporation, New York, 1962).

[11] T. P. Loftus, B. Petree, and J. T. Weaver, Radiology 86, 149 (1966).

[12] G. Mie, Ann. Phys. Lpz. 13, 857 (1904).

[13] T. E. Burlin and S. R. Husein, J. Sci. Instr. 42, 800 (1965).

[14] R. T. Berger, Radiation Res. 15, 1 (1961).

[15] T. P. Loftus and J. T. Weaver (private communication).

[16] S. I. Tomkeieff, Nature 155, 24 (1945).

(Paper 71C1-440) 